Please quote as: Kipp, P. \& Leimeister, J. M. (2013): Kollaborative Verbesserung von Ideen auf Web-basierten Ideenplattformen. In: Mensch \& Computer 2013 - Leichtgewichtige Werkzeuge zur Unterstützung von Kooperation und persönlichem Wissensmanagement, Bremen, Germany. 


\title{
Kollaborative Verbesserung von Ideen auf Web-basierten Ideenplattformen
}

\author{
Philipp Kipp ${ }^{1}$, Jan Marco Leimeister ${ }^{1}$ \\ Wirtschaftsinformatik, Universität Kasse ${ }^{1}$

\section{Zusammenfassung}

Lediglich ein Bruchteil der Ideen auf web-basierten Ideenplattformen kann von Firmen ausgewertet und umgesetzt werden. Das wird insbesondere dadurch unterstützt, dass aktuelle Plattformen sehr gezielt für die Entwicklung von möglichst viel Inhalt durch viele Beteiligte entworfen werden. Um jedoch besser von den Ideen der Plattformnutzer profitieren zu können, ist eine stärkere Fokussierung auf die Qualität der generierten Ideen notwendig. Dieser Beitrag demonstriert, wie Benutzerkollaboration auf web-basierten Ideenplattformen unterstützt werden kann. Das vorgestellte Kollaborationswerkzeug versetzt motivierte Teilnehmer in die Lage gemeinsam an der Verbesserung der bereits existierenden Ideen mitzuwirken. Dabei durchlaufen Sie einen strukturierten Prozess, der für beliebige Ideen wiederholbar abgebildet wird.

\section{Forschungshintergrund}

Web-basierte Ideenplattformen (WBIP) sind etablierte Werkzeuge zur groß angelegten Ideenfindung. WBIPs ermöglichen es Unternehmen und anderen Organisationen nicht nur, externe Stakeholder anzusprechen, sondern diese auch gezielt zur Ideengenerierung zu nutzen (Füller, Bartl et al. 2006; Reichwald \& Piller 2009; Bretschneider 2012). Nach Armstrong und Hagel (1995) fallen WBIP in die Kategorie der interessenorientierten (,,interest oriented“) virtuellen Communities. Sie dienen also dem Austausch der Nutzer über ein Thema von gemeinsamem Interesse. Dies können zum Beispiel Produkte und Dienstleistungen, aber auch soziale Fragestellungen sein. Bisherige Forschungsergebnisse zeigen, dass externe Stakeholder - und hierbei insbesondere Kunden - willens und in der Lage sind, ihre Ideen nicht nur untereinander auszutauschen und zu diskutieren, sonder sie auch mit Unternehmen und Organisationen teilen (Bretschneider \& Leimeister 2011). Aus der Kreativitätsforschung ist zudem bekannt, dass Nutzer von IT-Systemen in der Entwicklung kreativer Artefakte unterstützt werden müssen, um die besten Ergebnisse zu erzielen(Shneiderman 2002). Der Großteil der aktuellen WBIPs zielt allerdings vor allem auf die Generierung einer hohen Anzahl von Ideen ab und bietet nur rudimentäre 
Funktionalitäten, die auf die Unterstützung der Nutzer bei der Generierung von möglichst guten und umsetzbaren Ideen abzielen. Dies mag zwar für eine erfolgreiche Web-basierte Ideenfindung notwendig sein, scheint aber für die Entwicklung hochwertiger Ideen nicht hinreichend (Kipp, Wieck et al. 2013).

\section{Kollaborationsprozess}

Das in diesem Beitrag vorgestellte Artefakt ist ein Tool-gestützter Kollaborationsprozess für Web-basierte Ideenplattformen. Dieses sozio-technische System wird unter Verwendung der Design-Science-Methode entwickelt und evaluiert.

Der Kollaborationsprozess wird für existierende WBIPs entwickelt und fügt sich hinter die erste Phase des Innovationsprozesses, der Ideengenerierung, in die Konzepterarbeitung ein. Diese Positionierung ermöglicht es, ausgewählte und vielversprechende Ideen, die auf der WBIP generiert wurden, in den Prozess zu geben und kollaborativ weiter zu entwickeln. Hierbei kann der Plattformbetreiber als „Gatekeeper“ wirken und sich so in die Vorauswahl der Ideen einbringen (Heiskanen \& Similä 1992).

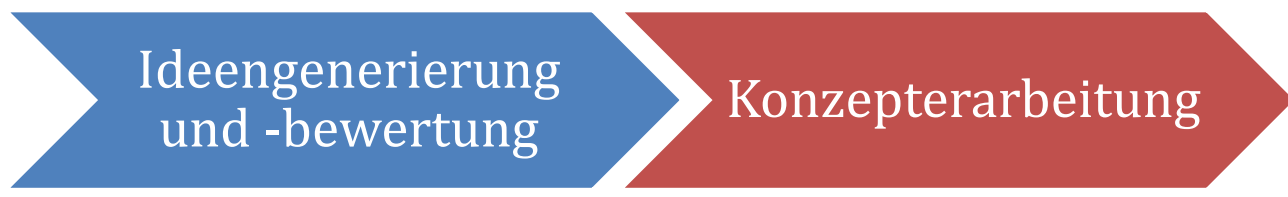

Abbildung 1:Eingliederung des Prozesses in die frühen Phasen der Innovationsentwicklung (Verworn \& Herstatt 2007)

Die besondere Herausforderung in der Übertragung von Kollaborationsprozessen auf webbasierte Plattformen liegt in den Besonderheiten der Web-basierten Kommunikation. Diesen wird insbesondere Rechnung getragen, indem die Benutzer jederzeit die Möglichkeit haben durch kleine Beiträge zu klaren Aufgabenstellungen zur Verbesserung der Ideen beizutragen. Der Ablauf des Kollaborationsprozesses wurde so entworfen, dass er nicht mit einer vordefinierten Gruppe von Teilnehmer abläuft. So kann jeder Nutzer der jeweiligen Ideenplattform jederzeit in den Prozess einsteigen und zur Weiterentwicklung der Idee beitragen. So stellt sich die Teilnahme für jeden potenziellen Teilnehmer sehr einfach dar. Um dieses Ziel zu erreichen wurde der Prozess in kleine, verteilte Arbeitspakete aufgeteilt und stellt sämtliche Beiträge und Zwischenergebnisse jederzeit für alle Nutzer der Plattform transparent dar.

Die dargestellte Version des Kollaborationsprozesses beruht auf sechs Schritten, die mit der initialen Idee beginnen und mit der gemeinsam ausgearbeiteten Idee enden. Die methodischen Grundlagen für die Entwicklung des im Werkzeug abgebildeten Kollaborationsprozesses orientieren sich am "Collaboration Process Design Approach“ (COPDA) nach Kolfschoten und De Vreede (Kolfschoten \& De Vreede 2009). 
Der erste Schritt, die „Problemdefinition“, ist die Analyse der Ausgangsidee mit dem Ziel, das ihr zugrunde liegende Problem zu identifizieren. Das erledigen die Teilnehmer, indem sie, basierend auf der initialen Idee, einzelne Aspekte des Problems identifizieren und mit den anderen Nutzern der Plattform teilen. Dies wird fortgesetzt, bis über einen bestimmten Zeitraum von den Nutzern keine Veränderungen mehr vorgenommen werden.

Im zweiten Schritt, der „Problemabstimmung“, geht es um die Relevanzabschätzung der zuvor generierten Problemaspekte. Die Aufgabe der Teilnehmer besteht hierbei darin, die Problembeschreibung zu kommentieren und zu bewerten. So kann das Meinungsbild der Community zu den Beiträgen für die weitere Bearbeitung schnell erfasst werden.

Die Prozessschritte drei und vier („Lösungsdefinition“ und „Lösungsabstimmung“) laufen analog zu den ersten zwei Prozessschritten ab, befassen sich aber mit der Lösung des zuvor ausgearbeiteten Problems und gehen dabei von der Lösung aus, die in der initialen Idee beschrieben wurde.

Schritte fünf und sechs („Problem-,, bzw. „Lösungsformulierung“) dienen der Aufarbeitung der bewerteten Brainstormbeiträge. Ziel dieser Prozessschritte ist die Ausformulierung einer gemeinsamen Beschreibung der Überarbeiteten Idee. Hierzu greifen die Teilnehmer auf sämtliche Ergebnisse der vorrangegangenen Prozessschritte (Beiträge und deren Bewertungen) zurück und nutzen eine kollaborative Textverarbeitung, die es erlaubt, dass mehrere Teilnehmer gleichzeitig am Text arbeiten. So entsteht eine gemeinsam überarbeitete, lesbare Version der Idee, die die kreativen Lösungen und unterschiedlichen Sichtweisen der Teilnehmer vereint.

Dieser sechs-stufige Prozess wurde mit drei verschiedenen Gruppen getestet, deren Aufgabe darin bestand, bestehende Ideen zur Verbesserung der universitären Lernumgebung weiterzuentwickeln. Die ausgearbeiteten Ideen wurden Experten vorgelegt, welche die Qualität der Ideen und insbesondere ihren Ausarbeitungsgrad nach dem Prozess signifikant höher bewerteten als davor.

\section{Software Artefakt}

Der beschriebene Kollaborationsprozess wurde in eine eigens entwickelte web-basierte Ideenplattform integriert. Die Implementierung des Kollaborationsprozesses als webbasiertes Werkzeug basiert auf dem CMS System Drupal 6 und wurde dort als eigenständiges und auf andere Plattformen übertragbares Modul implementiert. Dies ermöglicht eine Evaluation des Kollaborationsprozesses in Laborexperimenten und Feldtests mit verschiedenen Teilnehmergruppen. Die Ergebnisse der Sitzungen und Experimente werden dann als Grundlage für die zukünftige Überarbeitung des Tools dienen.

Die Prozessschritte zur Identifikation und Konkretisierung von Problem und Lösung aus denen sich die Ideen zusammensetzen wurden in Form eines Brainstorms implementiert, zu dem jeder Benutzer der Plattform zeit- und ortsunabhängig beitragen kann. Um die Einschätzung der Relevanz der einzelnen Brainstormingbeiträge zu gewinnen können diese 
während dieser Prozessschritte von den Prozessteilnehmern bewertet werden. Dies versetzt die Teilnehmer in die Lage, die Relevanz der kollaborativ zusammengetragenen Problemund Lösungsaspekte einzuschätzen und in der Ausformulierung entsprechend zu Berücksichtigen. Zur Unterstützung der Prozessschritte für die Formulierung von Problem und Lösung als zusammenhängende Texte wird die kollaborative Textverarbeitung Etherpad Lite $^{1}$ eingesetzt. Dieser erlaubt es den Teilnehmern jederzeit gleichzeitig zur Formulierung beizutragen und gibt den Forschern die Möglichkeit, den Entstehungsprozess der überarbeiteten Idee detailliert nachzuvollziehen, indem jede Nutzereingabe einzeln nachvollziehbar ist.

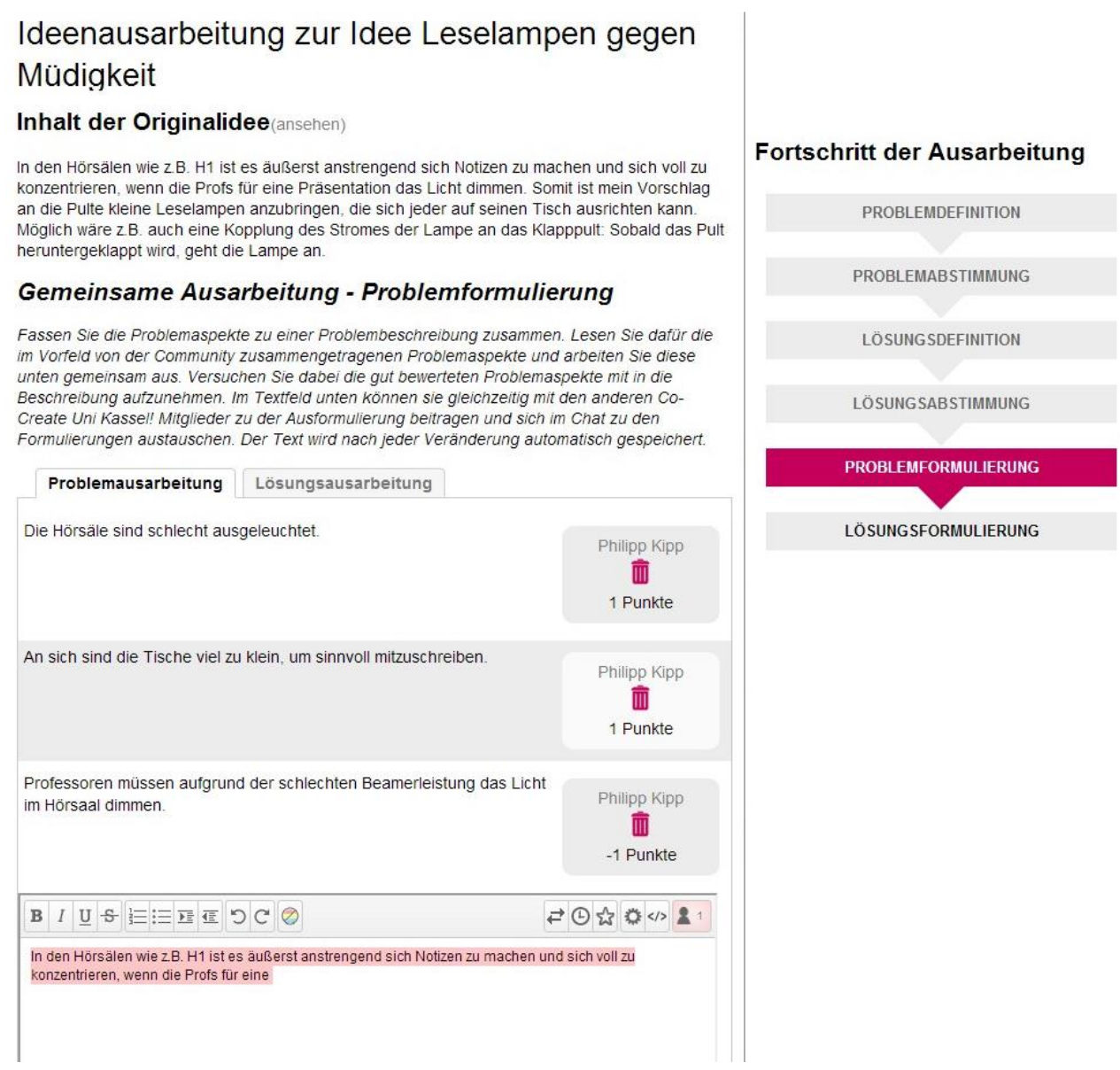

Abbildung 2: Bildschirmfoto des Kollaborationstools mit einer Beispielidee

\footnotetext{
${ }^{1}$ http://www.etherpad.org
} 
Während des gesamten Bearbeitungsprozesses sind alle bisherigen Arbeitsergebnisse sowie die initiale Idee für die Bearbeiter einsehbar. So ist sichergestellt, dass die Bearbeitungsschritte auf einander aufbauend bearbeitet werden können. Abbildung 1 zeigt ein beispielhaftes Bildschirmfoto des Kollaborationswerkzeugs, das in die Ideenplattform „Co-Create Uni Kassel!“ eingebunden ist.

\section{Ausblick}

Dieser Beitrag zeigt ein Kollaborationswerkzeug, das es Nutzern von web-basierten Ideenplattformen ermöglicht gemeinsam ausgewählte Ideen auf der Plattform zu verbessern. Erste Endnutzertests mit Expertenbewertung konnten zeigen, dass die Ideenqualität durch den eingesetzten Prozess verbessert werden kann. Evaluationen mit vielen Teilnehmern und Versuche stehen aktuell noch aus und sind in den nächsten Monaten geplant. Durch die Integration des Werkzeugs in eine bestehende Ideenplattform kann die Evaluation sehr praxisnah mit echten Nutzern, aber auch in kontrollierten Laborumgebungen erfolgen. Diese sollen Rückschlüsse auf Prozessanpassungen und die Eignung des gewählten Collaboration Engineering Ansatzes für die Entwicklung von werkzeugunterstützen verteilten Kollaborationsprozessen liefern. So soll das Kollaborationswerkzeug dazu beitragen, die Kundenintegration über web-basierte Ideenplattformen erfolgreicher im Sinne einer größeren Anzahl qualitativ hochwertiger und umsetzbarer Ideen zu gestalten.

\section{Schlussteil}

\section{Kontaktinformationen}

Philipp Kipp: $\quad$ philipp.kipp@uni-kassel.de

Jan Marco Leimeister: leimeister@uni-kassel.de

Universität Kassel, Wirtschaftsinformatik, Pfannkuchstr. 1, 34121 Kassel

\section{Literaturverzeichnis}

Armstrong, A. G. \& Hagel, J. (1995). The real value of online communities. Creating value in the network economy. Boston, MA, USA: Harvardn Business Review Book Series, 173-185.

Bretschneider, U. (2012). Die Ideen Community zur Integration von Kunden in die frühen Phasen des Innovationsprozesses: Empirische Analysen und Implikationen für Forschung und Praxis. Wiesbaden, Springer Gabler.

Bretschneider, U. \& Leimeister, J. M. (2011). Getting costomers' motives: Lean on motivation theor for designing virtual ideas communities. Governance and Sustainability in Information Systems: Managing the Transfer and Diffusion of IT, Proceedings International Working Conference IFIP, Hamburg, Nüttgens, M.; Gadatsch, A.; Kautz, K.; Schirmer, I.; Blinn, N. 
Füller, J., Bartl, M., Ernst, H. \& Mühlbacher, H. (2006). Community based innovation: How to integrate members of virtual communities into new product development. Electronic Commerce Research 6(1), 57-73.

Heiskanen, A. \& Similä, J. (1992). Gatekeepers in the action structure of software contracting: a case study of the evolution of user-developer relationships. Proceedings of the 1992 ACM SIGCPR conference on Computer personnel research, ACM.

Kipp, P., Wieck, E., Bretschneider, U. \& Leimeister, J. M. (2013). 12 Years of GENEX Framework: What did Practice Learn from Science in Terms of Web-Based Ideation? 11. Internationale Tagung Wirtschaftsinformatik, Leipzig.

Kolfschoten, G. L. \& De Vreede, G.-J. (2009). A Design Approach for Collaboration Processes: A Multimethod Design Science Study in Collaboration Engineering. Journal of Management Information Systems 26(1), 225-256.

Reichwald, R. \& Piller, F. (2009). Interaktive Wertschöpfung - Open Innovation, Individualisierung und neue Formen der Arbeitsteilung. Wiesbaden, Gabler.

Shneiderman, B. (2002). Creativity Support Tools. Communications of the ACM 45(10), 116 $-120$.

Verworn, B. \& Herstatt, C. (2007). Bedeutung und Charakteristika der frühen Phasen des Innovationsprozesses. Management der frühen Innovationsphasen: Springer, 3-19. 